\title{
le glissement du Friolin, en Savoie : un mouvement majeur révélé par les photographies
}

\author{
the Friolin rockslide in Savoy: \\ major movement revealed by photos
}

\section{Bernard GOGUEL*}

Rev. Franç. Géotech. n 48 , pp. 55-64 (juillet 1989)

\section{Résumé}

La dissolution progressive d'un soubassement de gypse désorganise la montagne du Friolin, surveillée depuis les années soixante. D'abondantes chutes de blocs s'y produisirent de 1982 à 1984, sur une surface de 20 hectares.

L'étude comparative des photographies prises au fil des années montre que le versant rocheux accidenté est descendu en bloc de plus de $50 \mathrm{~m}$, dans ce qui apparaît comme un glissement rotationnel d'environ 10 millions de $\mathrm{m}^{3}$.

Ce mouvement d'ampleur exceptionnelle s'est produit en quelques années, et nul ne sait s'il est véritablement arrêté. La photogrammétrie pourrait en préciser la cinématique, pour éclairer l'estimation des risques associés et guider l'organisation de la surveillance.

\footnotetext{
Abstract

Progressive dissolution of underlying gypsum is disturbing Mount Friolin on which an eye has been kept since the $1960 \mathrm{~s}$. From 1982 to 1984 there were a large number of rock falls over an area of 20 hectares.

Comparison of photos taken from known points on the ground year after year shows that the slope in question has dropped coherently more than $50 \mathrm{~m}$ in a circular slide of about 10 million cubic metres.

This movement of exceptional magnitude has occurred over a period of several years and nobody knows if it has stopped completely. Photogrammetry could give details on the kinematics of the slide so as to better assess the risks involved and orient monitoring.
} 


\section{CONTEXTE GÉNÉRAL}

La pointe de Fríolin, à l'extrémité nord-ouest du massif de Bellecôte, domine de $1200 \mathrm{~m}$ la vallée de Peisey-Nancroix (Savoie). Elle recèle une menace, signalée de longue date par Jean GOGUEL, d'éboulement majeur susceptible de venir couper la route d'accès à Rosuel (porte du Parc National de la Vanoise) entre le Palais de la Mine et les Lanches.

Voir carte IGN $1 / 25000^{\circ}$ Moutiers (3533) Est, ou assemblage touristique 235 Massif de la VanoiseTarentaise, et profil (fig. 1).

C'est un ensemble de roches rudes (" gneiss " du socle métamorphique carbonifère de la Vanoise), culminant sous la forme d'un petit plateau vers la cote 2700 , dont le flanc Est constitue un escarpement de pente moyenne $45^{\circ}$ sur $250 \mathrm{~m}$ de dénivellation et $600 \mathrm{~m}$ de longueur (sens N-S). Il repose sur une grande épaisseur, de l'ordre de $500 \mathrm{~m}$, de terrains solubles et peu résistants : gypse et cargneules. Le niveau du contact gneiss/gypse n'est pas exactement connu.

La solubilité du gypse est une cause d'instabilité pour les masses rocheuses susjacentes, surtout après qu'une première fissuration a rendu le gneiss perméable. La morphologie de la montagne témoigne de tassements et d'adaptations de la masse rocheuse: deux failles d'áge quaternaire (J. GOGUEL, 1969) coupent le versant Sud, en y relevant le bas d'une pente absolument régulière déterminée par un manteau d'éboulis; de grands décrochements marquent le plateau sommital, formant des escarpements concentriques avec des basculements en échelon emboîtés (fig. 2).

Tout cela est saisissant sur photos aériennes. Au sol, le plateau apparaît intensément crevassé et fissuré sur plusieurs hectares, avec des indices de fraîcheur des mouvements (déchirures de la terre végétale) qui avaient attiré l'attention au début des années soixante. Une dizaine de repères de fortune, simples marques de peinture appliquées en 1961, témoignèrent pendant quelques années d'ouvertures de l'ordre du centimètre par an (pour des crevasses larges de 0,5 à $1 \mathrm{~m})$. Puis le mouvement parut se ralentir... et les marques s'effacèrent.

\section{POSITION DU PROBLÈME}

Le caractère progressif de la dissolution du gypse explique une évolution longue (à l'échelle humaine s'entend) : une tranche d'eau de $1 \mathrm{~m}$, ordre de grandeur des précipitations annuelles qui s'infiltrent en totalité dans les crevasses béantes du plateau, peut dissoudre $1 \mathrm{~mm}$ de gypse.

On conçoit que l'évolution puisse se faire par à-coups, avec des réajustements successifs au fil de la progression des dissolutions et des dislocations induites. On ignorait il y a encore quelques années la forme que prendrait cette évolution : série de petites adaptations locales, grands tassements verticaux comme ceux ayant induit les failles du versant Sud, basculement et effondrement du haut de la falaise, ou glissement plus ou moins généralisé du versant susceptible de se transformer en éboulement après dislocation de la masse rocheuse?

Si une masse rocheuse déstabilisée devait s'ébouler un jour, il est possible dans un premier temps - ou pour une première fraction, de quelques millions de $\mathrm{m}^{3}$ - qu'elle s'arrête au bourrelet de terrain (ancienne moraine) qui domine les chalets d'alpage situés à la cote 1992. Mais si toute la masse fissurée partait à la fois, elle pourrait dépasser cette zone de recueil du Grand Plan.

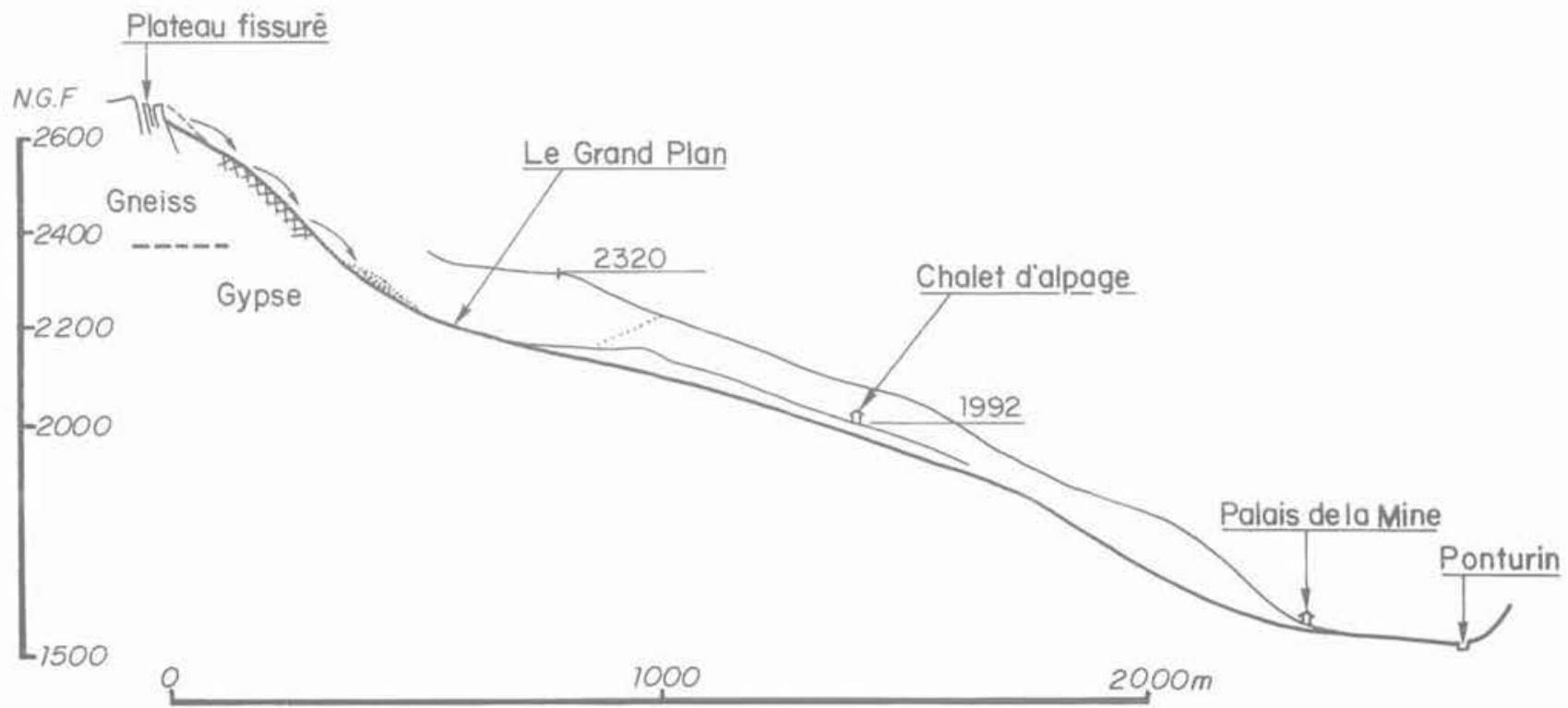

Fig. 1. - Friolin, profil général orienté vers le Nord-Est.

Fig. 1. - General NE-SW profile. 


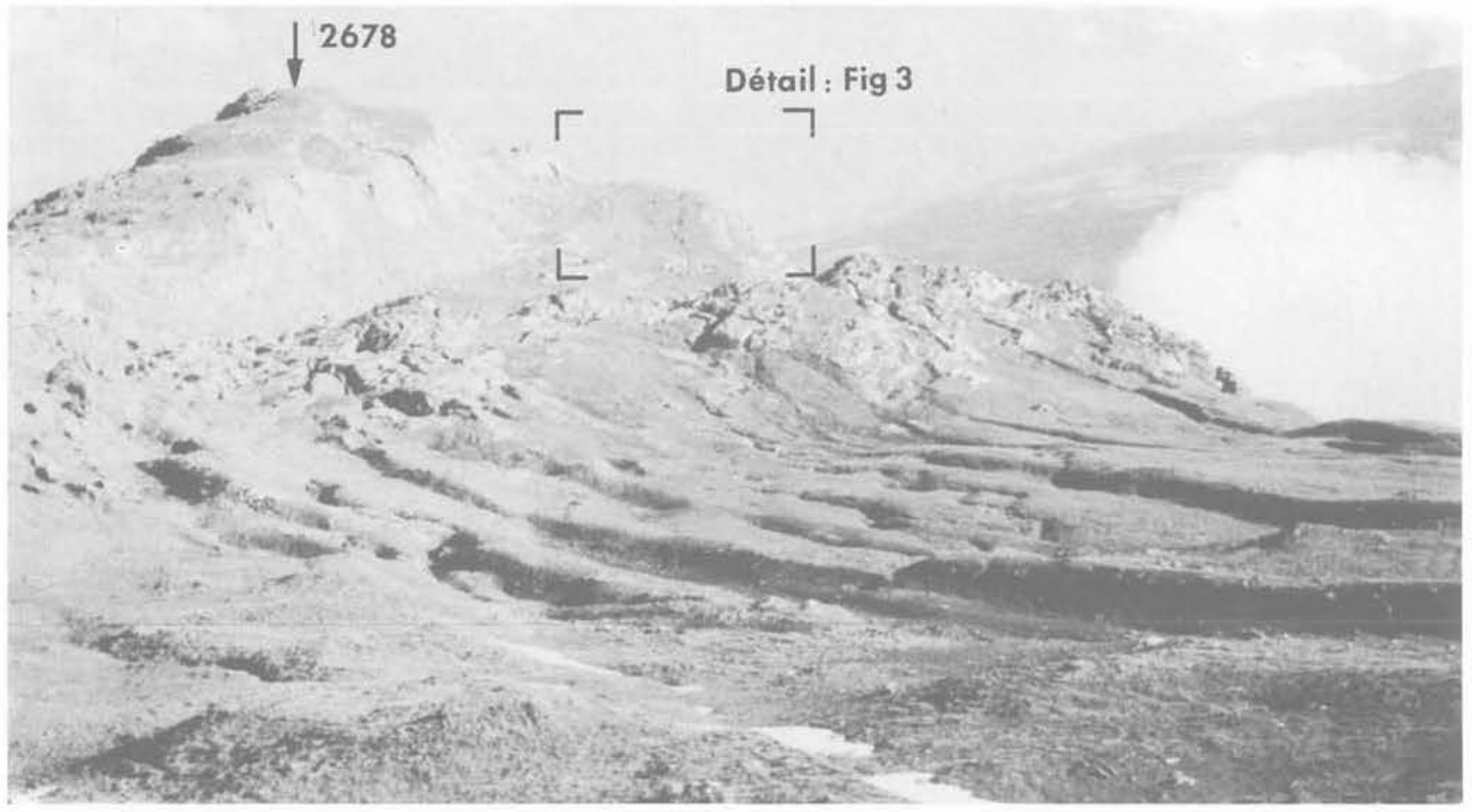

Fig. 2. - Plateau sommital fissuré (vu du sommet sud, $2739 \mathrm{~m}$, vers le nord; cliché 1964).

Fig. 2. - Crazed summit plateau (seen from south top, $2739 \mathrm{~m}$, looking north: 1964 photo).

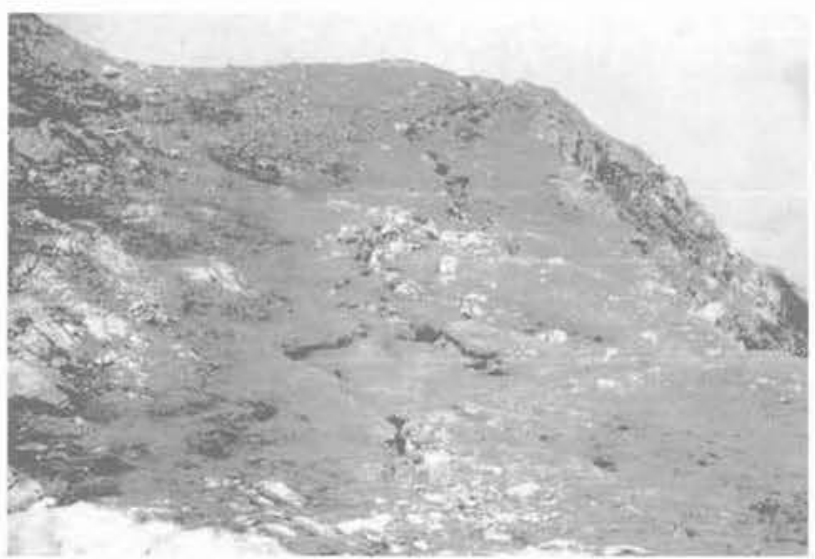

a) en 1971

a) in 1971

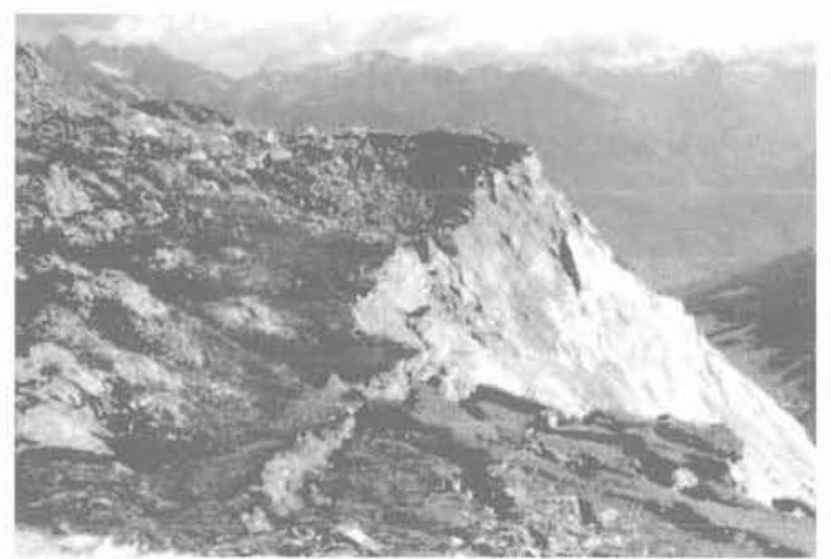

b) en 1987

b) in 1987

Fig. 3. - Détail Nord-Est du plateau sommital

Fig. 3. - Detail of N.E. corner of summit plateau

L'observation montre en effet que les très grands éboulements coulent littéralement sur les pentes, selon une dynamique qui n'a plus aucune similitude avec celle des chutes de blocs isolés ou des écroulements de petites falaises. Dans cette hypothèse extrême, le fond de la vallée de Peisey serait atteint dans une zone vitale pour son activité touristique estivale.
Les zones exposées aux différents degrés de risque ont été indiquées dans un document qui préfigurait les cartes ZERMOS (J. GOGUEL et M. HUMBERT, 1972). L'approche du danger se signalerait sans doute par de nombreuses chutes de blocs isolés sur le versant du Friolin dominant l'alpage. 


\section{LA CRISE DE 1982}

Au printemps et durant tout l'été 1982, le Friolin connut une "crise " marquée par d'abondantes chutes de blocs dans son versant Est. Ce fut une dégringolade quasi permanente sur un front large de plus de $600 \mathrm{~m}$, qui vint consteller les pentes supérieures de l'alpage de grosses pierres nouvelles. Quelques blocs de plusieurs $\mathrm{m}^{3}$ dépassèrent même le sentier du Lac de l'Etroit, $500 \mathrm{~m}$ sous le sommet.

L'attention fut nettement attirée, et l'affaire observée par les quelques bergers qui en avaient une vue directe (soit de l'alpage situé juste en dessous, soit de la montagne d'en face) ou par les chasseurs de chamois de passage. Mais les montagnards sont habitués aux chutes de blocs et aux avalanches; assez vite, le phénomène * était devenu normal, on n'y faisait même plus attention ». Et la montagne est si vaste alentour, qu'il faut s'intéresser de près au versant accidenté pour saisir ses véritables dimensions.

Fin juillet 1982, il tombait à peu près un bloc toutes les minutes. Cette année là, les bêtes ne furent pas montées au Grand Plan, zone de recueil des blocs qui allèrent le plus loin, avant le mois de septembre.

Les témoignages restent imprécis en ce qui concerne l'intensité et la durée du phénomène: l'affaire se passe en haute montagne, couverte de neige les deux-tiers de l'année, dans un endroit peu fréquenté : et l'alpage (communal) changea plusieurs fois d'occupant au début des années 80 . Quelques observations ponctuelles pourraient faire remonter les prémices de cette crise à l'été 1981, mais c'est apparemment à la fonte des neiges 1982 que les éboulements prirent un tour exceptionnel. L'activité du versant s'est poursuivie en 1983 et 1984, moins intense cependant. Il tombait encore quelques blocs de temps en temps en 1986 et 87 , presque plus durant l'été 88 .

L'aspect du versant Est du Friolin fut visiblement changé par cette crise. Toute la bordure du plateau sommital s'est effondrée, laissant apparaître un arrachement continu sur plus de $500 \mathrm{~m}$ de largeur (voir fig. $3 a$ et $b$, ou $4 a$ et $b$ ). De nombreux blocs ont dévalé les couloirs de l'escarpement rocheux, engraissant quelques anciens éboulis et, plus visiblement, en générant de nouveaux très étendus. Les gros blocs sont descendus assez bas dans les pentes d'herbe qui s'adoucissent vers $2150 \mathrm{~m}$ d'altitude, $500 \mathrm{~m}$ sous le sommet, roulant et rebondissant puis terminant leurs courses par des glissades en labourant le manteau végétal. De grands arrachements se sont développés dans les pentes d'herbe raides sous l'escarpement, avec des signes de glissements locaux sur un ou deux hectares vers le milieu de la zone bombardée.

La Direction de la Sécurité Civile fut alertée et le géologue du BRGM appelé en renfort, spécialiste des chutes de blocs et risques d'éboulements, put compléter les visites au sol par une reconnaissance héliportée début septembre.

Malgré tous les désordres qui l'entouraient, l'escarpement rocheux médian était pratiquement intact et conservait son apparence habituelle, faite d'une succession d'éperons et de couloirs plus ou moins herbus.
La complète intégrité et la conservation de la cohérence du versant sur près d'une quinzaine d'hectares (avec seulement quelques desquammations très locales) pouvait laisser croire à l'absence de déplacement d'ensemble, même s'il était patent que la roche * travaillait $»$. Les observateurs restaient dans le doute quant à la forme exacte du mouvement d'adaptation de la montagne, générateur des chutes de blocs (J. GOGUEL et A. PACHOUD, 1982).

Par la suite les chutes de blocs s'espacèrent et, même si elles ne cessèrent pas d'un coup, on considéra que la crise était passée. Elle s'était traduite par plusieurs accumulations nouvelles de blocs sous la falaise, totalisant de l'ordre de $200000 \mathrm{~m}^{3}$ pour fixer les idées. A raison de 1 à $10 \mathrm{~m}^{3}$ par bloc tombé, cela en représente un bon nombre.

\section{L'APPORT DES PHOTOGRAPHIES}

\subsection{Constat principal}

Une vue d'ensemble du versant Est du Friolin avant la crise, prise en 1976 du sommet de l'Aiguille Grive (altitude $2732 \mathrm{~m}$, à $5,2 \mathrm{~km}$ de distance), nous a été laissée par Jean GOGUEL. La figure 4a reproduit un petit extrait de la diapo correspondante, dont il ne disposait pas durant l'été 1982. La photo reproduite ici en pleine page (fig. 4b) fut prise par lui du même

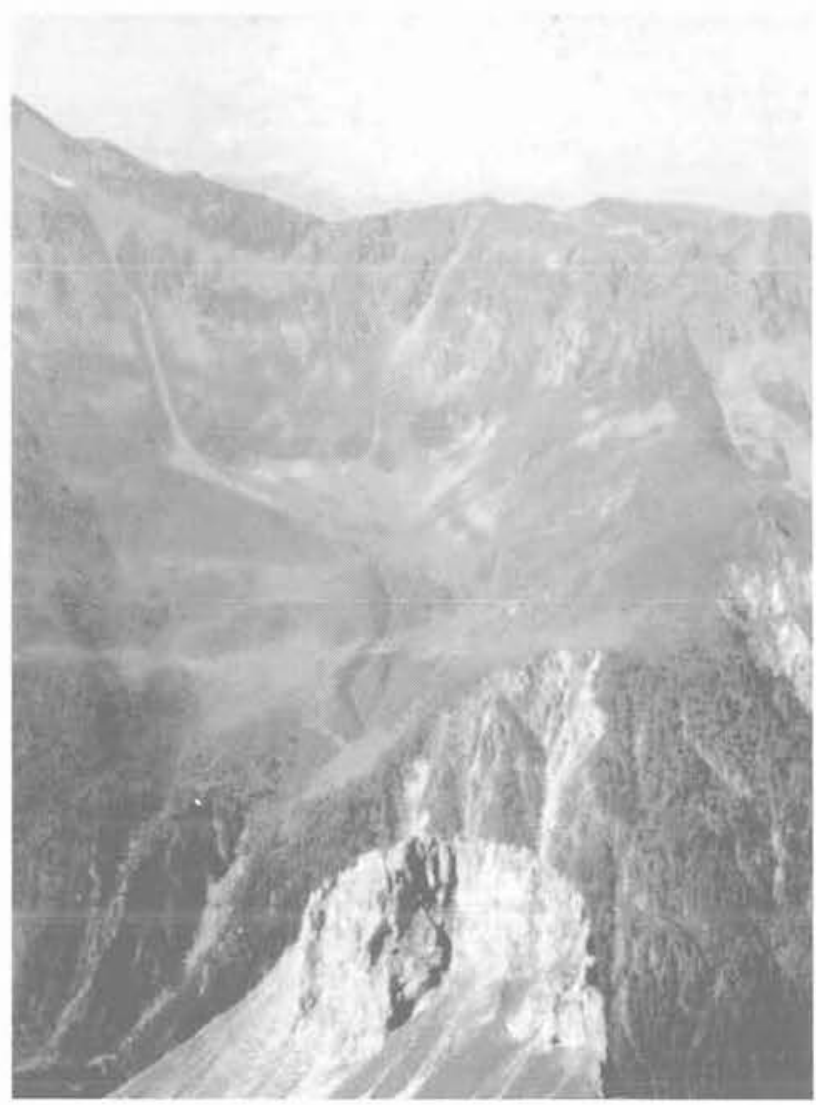

Fig. 4. - Versant Est du Friolin vu d'un même point éloigné : a) en $1976 \quad$ b) en 1983 (ci-contre).

Fig. 4. - East face of Frolin, from the same point: $\begin{array}{ll}\text { a) in } 1976 & \text { b) in } 1983 \text { (opposite). }\end{array}$ 


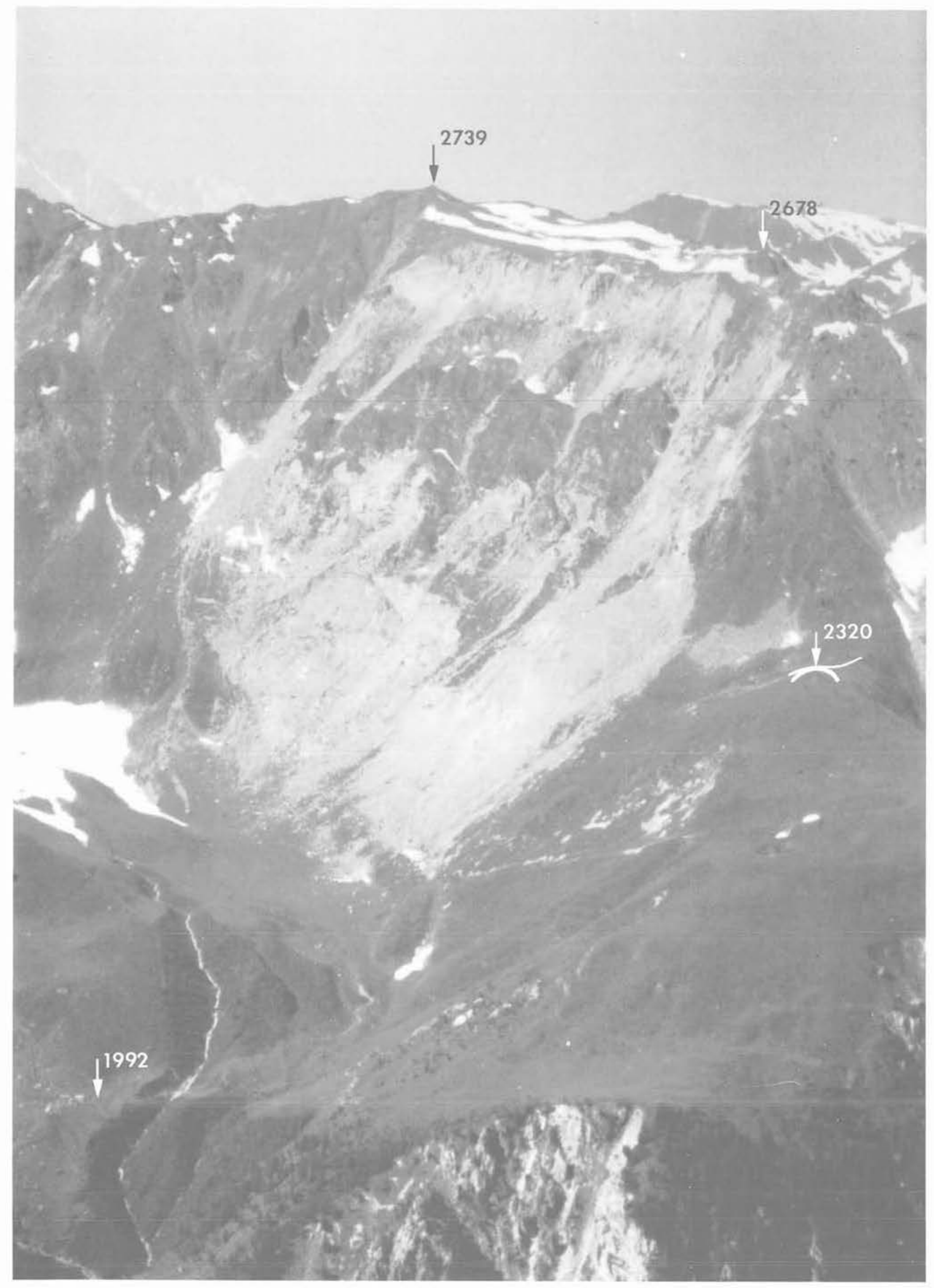


point en 1983 ; mais c'est d'un autre point plus aisément accessible (en haut du plateau du Rey, vers $2300 \mathrm{~m}$ d'altitude et à $4,5 \mathrm{~km}$ de distance du Friolin) qu'il a institué à partir de juillet 1982 une surveillance photographique systématique du versant accidenté, avec des photos annuelles en couleur, tant d'ensemble que de détail, qui constituent un précieux témoignage de l'évolution du versant accidenté pendant et après la crise.

Des photos comparatives prises localement sur le plateau sommital du Friolin sont également disponibles, qui permettent d'apprécier exactement le recul sa de bordure Est effondrée (20 à $40 \mathrm{~m}$ selon les endroits, un peu moins aux extrémités : cf. fig. 3) et d'observer qu'à part cela les crevasses et fissures éloignées du bord ont gardé une apparence pratiquement inchangée à 25 ans d'intervalle.

On dispose des photos aériennes systématiques de l'IGN prises en 1948, 1956, 1967, 1970, 1972, 1980 (avant la crise), puis le 22 aout 1982 (indisponibles à l'époque du diagnostic effectué sur le champ, et même insoupçonnées puisqu'en avance sur la périodicité normale de 5 ans : ces photos furent prises pour l'Inventaire Forestier National) et le 1er septembre 1986. Elles offrent l'avantage de la vision stéréo, mais le point de vue est changeant d'une année à l'autre, ainsi que l'échelle et parfois même le sens de défilement (E-W, ou N-S).

Des heures ont été passées à examiner et comparer ces nombreuses photos, pour s'imprégner à la fois des structures d'ensemble et des moindres détails susceptibles de permettre des rattachements. La grande qualité de la carte IGN aide à situer les repères, et à trouver des échelles. Une bonne connaissance du terrain, approché à pied à plusieurs reprises et observé sous tous les angles et à toutes les distances possibles, aide à mémoriser ce gigantesque puzzle en s'affranchissant des variations d'éclairage.

Et soudain le déclic s'est fait, révélation formidable aisément vérifiée en remontant à l'Aiguille Grive (photo de contrôle 1988, peu différente de la photo 1983 reproduite ici en fig. 4b) et qui pourra paraître une évidence au lecteur prévenu : les escarpements rocheux du flanc Est du Friolin dans leur ensemble, sur toute leur hauteur de $250 \mathrm{~m}$ en dessous du plateau sommital, sont descendus en un seul bloc sur un front large de plus de $500 \mathrm{~m}$, accusant une dénivellation parfaitement mesurable malgré la rusticité des moyens employés : environ $55 \mathrm{~m}$ au total. Les photos aériennes montrent que leur pied s'est déplacé dans le même temps d'à peu près autant vers l'Est. Le déplacement global du versant, dans le sens de la pente $\left(45^{\circ}\right.$ en moyenne), avoisinerait donc $75 \mathrm{~m}$.

\subsection{Forme du mouvement}

Force est d'admettre qu'il y a eu une rupture en grand du versant, s'apparentant à un glissement de type rotationnel, depuis le couloir Sud issu du point 2739 jusqu'à l'arête Nord-Est qui est scalpée localement par la déchirure. Les chutes de blocs de 1982 ont été par- ticulièrement abondantes à ces deux bordures du glissement, où elles ont alimenté de nouveaux éboulis.

Certains arrachements préexistants observés dans les pentes d'herbe vers $2300 \mathrm{~m}$ d'altitude pourraient correspondre à des effondrements de dissolution du gypse, susceptibles d'avoir déstabilisé le versant de roches dures qui les domine. Mais d'autres arrachements vers la même altitude, notamment à l'aplomb du grand couloir Nord-Est, témoignent du réajustement des terrains meubles ou friables au débouché inférieur de la surface de rupture, qui induit un gonflement appréciable.

Celle-ci est forcément passée assez profondément, puisque la falaise a joué d'un seul bloc. Peut-être ya-t-il eu déformation continue du plateau supérieur, mais il suffit de faire passer une surface de rupture hypothétique par l'effondrement du bord - où les « miroirs » sont particulièrement nets - pour arriver à une estimation du volume glissé de l'ordre de 10 millions de $\mathrm{m}^{3}$ (fig. 5).

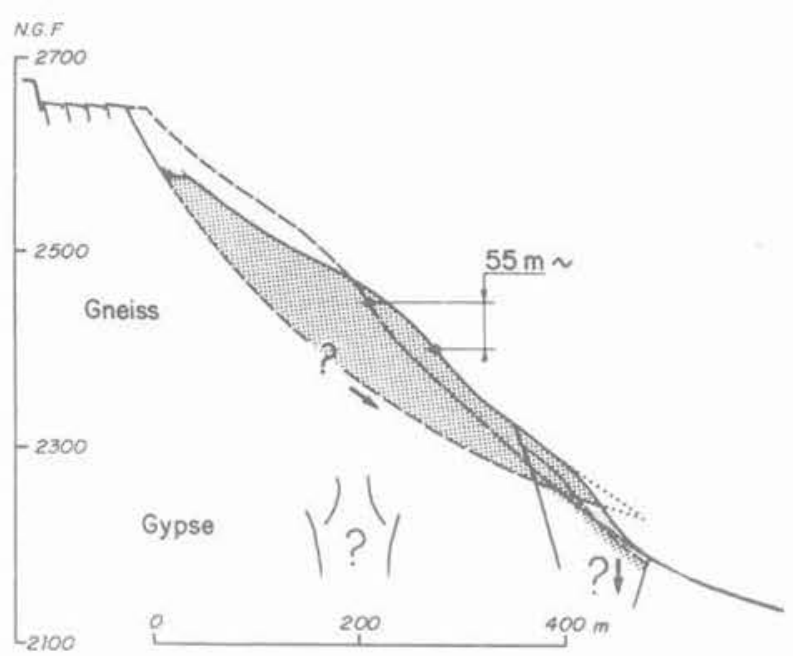

Fig. 5. - Coupe interprétative.

Fig. 5. - Interpretative cross-section.

Il faut tirer l'affaire au clair, pour essayer de savoir à quoi s'en tenir dans lavenir. Les techniques photogrammétriques et d'aérotriangulation pourraient être mises en cuvre pour décrire précisément les mouvements ayant affecté le plateau sommital et le versant, en en fournissant une cinématique détaillée. L'ampleur des déplacements est telle, qu'il suffirait d'exploiter les clichés aériens disponsibles réalisés en "chambres métriques » sans qu'il soit besoin d'envoyer aucune mission de géomètres au sol : les équipes de recherche et travaux spéciaux de l'IGN ont déjà traité avec succès des cas similaires, et annon. cent une précision meilleure que le mètre.

\subsection{Etude des photographies}

Toutes les vues disponibles, tant de détails que d'ensemble sous différents angles (fig. 6 , vue en montant à Bellecôte, par exemple), aident à appréhender l'état du Friolin. Mais les photos $24 \times 36$ répé- 


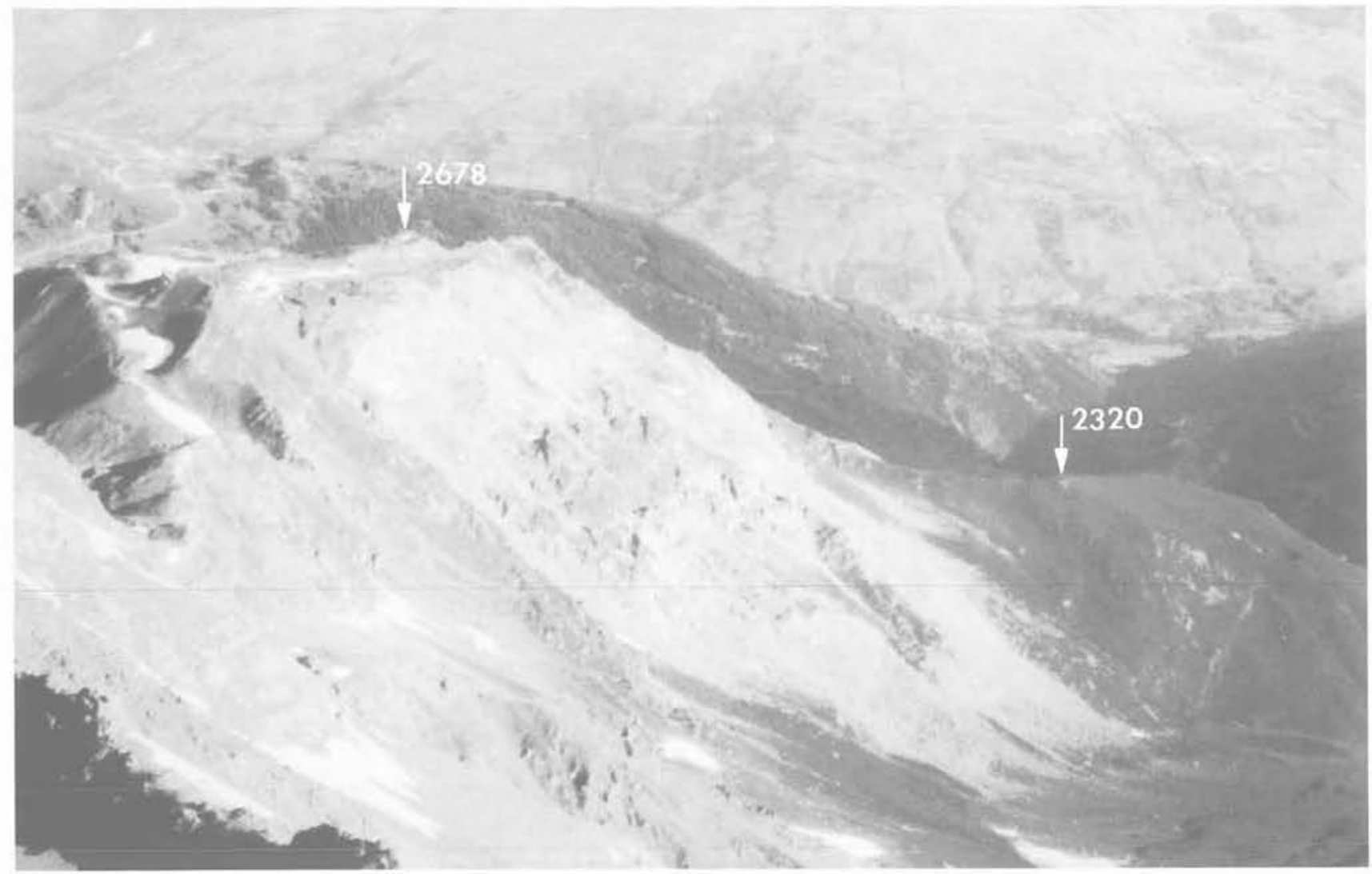

Fig. 6. - Le glissement en vue plongeante du Sud-Est, en août 1987 (remarquer les failles quaternaires qui décrochent le versant sud, sur la gauche de la photol.

Fig. 6. - The slide seen from above, looking north-west, in August 1987 (note the Quaternary faults scarring the south slope, on the left of the photol.

tées depuis des points éloignés connus sur les montagnes d'en face, d'altitudes équivalentes, constituent aussi un moyen simple d'accéder à la composante verticale du déplacement d'ensemble.

Le report sur transparents des principaux traits du versant étudié et de son environnement fixe a d'abord permis, en usant de couleurs différentes et en recherchant les meilleures superpositions à partir de photos prises strictement des mêmes points :

- de s'affranchir des différences d'éclairage et d'enneigement ;

- de constater combien l'escarpement rocheux de la face Est du Friolin a conservé toute sa cohérence depuis 1976, en dépit d'écaillages superficiels :

- d'apprécier l'ampleur du mouvement d'une prise de vue à lautre (avec une échelle d'altitudes aisée à établir d'après la carte) :

- de subodorer un possible écrasement du pied sud (où s'accumulent les pressions d'arrêt et se développent les desquammations les plus notables), à la limite de la précision pratique de détection du procédé.

Plus directement ensuite, le simple tirage des diapos agrandies sur négatifs transparents et leurs superpositions deux à deux ont permis un contrôle incontestable et une mesure immédiate des décalages verticaux, en même temps qu'une évaluation de la précision autorisée.
Le versant accidenté est vu de loin, d'une altitude voisine, sous un angle faible (à peine $5^{\circ}$ verticalement), encadré de quelques points cotés bien repérables. Pour déterminer la composante verticale du déplacement, une simple règle de trois suffirait si il n'y avait quelques distorsions.

La première distorsion tient à l'appareil, qui ne donne pas autant de millimètres par unité d'angle au centre et sur les bords de l'image. On s'en affranchit au mieux en cadrant strictement à l'identique (et pour cela, il faut partir en montagne avec les photos pré cédentes dans son sac !).

La seconde distorsion tient aux positions relatives des points examinés et du point de prise de vue, qui n'est pas à l'infini. Pour notre versant vu à 4,5 ou $5,2 \mathrm{~km}$ (depuis, respectivement, une altitude voisine de celle de sa base ou de son sommet), on s'est limité à déterminer la correction pour le détail le plus remarquable de la falaise, à partir duquel se retrouvent toujours les superpositions de l'ensemble. Soit un facteur de 1,15 ou 0,93 selon le point de vue.

\subsection{Alignements de surveillance}

Le versant accidenté est absolument impraticable. Mais on peut s'en approcher latéralement, et voir en enfilade les principaux éperons de la falaise surveillée. 
Un repère a ainsi été posé en 1982, au pied de l'arête Nord-Est du Friolin, d'où l'on voit cinq plans successifs dont le plus éloigné (à $800 \mathrm{~m}$ environ) se détache sur les pentes stables situées au-delà du couloir Sud.

Là comme ailleurs, les contraintes d'éclairage sont sévères pour disposer de photos exploitables. Et il faut bien ajuster les cadrages et les focales utilisées. Il est paradoxal que ces photos de surveillance, qui auraient dû être les premières à détecter l'ampleur du mouvement, aient été abandonnées après quelques années sans que la cible de visée à placer sur le fond y ait jamais été montée.

Leur exploitation est en fait assez délicate : il faut distinguer et clarifier les plans successifs, en tracer les formes caractéristiques sur transparents, et travailler par superpositions. Alors seulement, on voit s'esquisser le film colossal de l'écoulement du versant vu transversalement depuis sa rive gauche. La réalité des déplacements étant ainsi formellement confirmée. seule l'évaluation de leur ordre de grandeur est accessible à ce procédé : dès lors que les visées sont inclinées et en biais, le dépouillement quantitatif des photos devient vite inextricable. Mais un avantage particulier de ces photographies est leur grande finesse d'évaluation : le décalage observé entre 1982 et 1983 s'est trouvé accru d'un tiers entre 83 et 84 , et d'autant entre 84 et 88 .

\subsection{Chronologie}

Elle se résume par la figure $n^{\circ} 7$, exprimant le déca. lage altimétrique global du versant par rapport à l'état de référence 1976 (encore inchangé en 1980 d'après les photos aériennes, et sachant que les chutes de blocs témoignant du mouvement n'ont pas véritable. ment démarré avant l'automne 1981, sinon même plus probablement la fonte des neiges du printemps 1982). Le décalage atteignait environ $30 \mathrm{~m}$ à la fin juillet $1982,3 \mathrm{~m}$ de plus un mois plus tard, $45 \mathrm{~m}$ en 1983 ; il s'est accru ensuite de $5 \mathrm{~m}$ en 1984 , et $5 \mathrm{~m}$ supplémentaires entre 1984 et 1988 (n'ayant semblet-il pas évolué sensiblement en 1985 et 86 , mais les photos de ces années là ont des éclairages peu favorables).

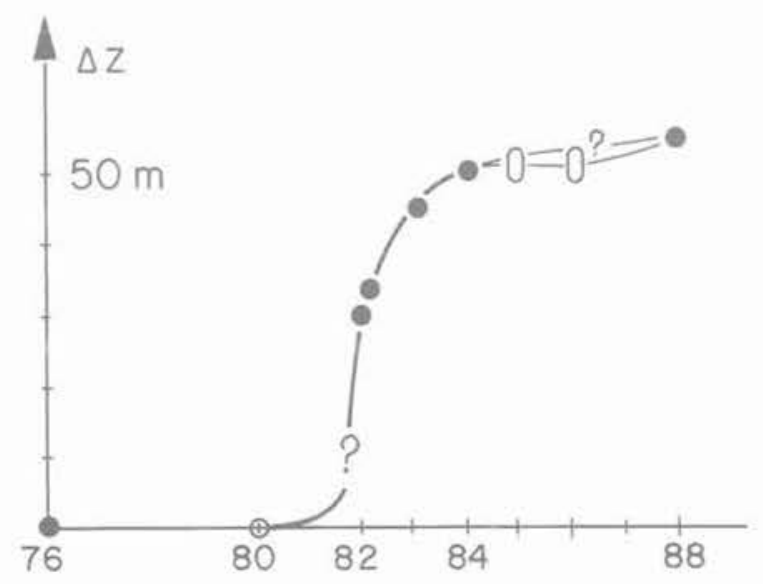

Fig. 7. - Dénivellation globale constatée. Fig. 7. - Observed change in level.
Au total, les $55 \mathrm{~m}$ entre 1976 et 1988 sont incontestables, comme les $20 \mathrm{~m}$ entre juillet 82 et la situation de 1984-85-86.

\subsection{Evolution du pied}

Le pied du versant accidenté comporte de grands arrachements, qui s'effritent et reculent par petits glissements successifs tout en étant battus par les chutes de pierres issues de la paroi rocheuse. Une partie d'entre eux sont probablement attribuables à des effondrements de dissolution du gypse, qui se seraient étendus dans les années précédant la crise de 82 .

Seules les photos d'ensemble permettent d'apprécier l'évolution de ce genre de choses. Là aussi, il est important de les prendre toujours des mêmes points et en cadrant sur l'environnement fixe.

Au fil des années, ces photos montrent aussi la progression du bourrelet comprimé qui raidit et disloque la prairie vers le Sud, là où le glissement a dâ trouver une certaine butée de pied du fait que les pentes supérieures du Grand Plan en dessous tournent vers l'Est. Cette zone critique est à surveiller tout particulièrement,

\section{INDICATIONS COMPLÉMENTAIRES}

\subsection{Structure du versant}

L'escarpement rocheux du versant Est du Friolin était constitué d'une succession d'éperons et de couloirs plus ou moins individualisés, entre les altitudes 2400 et $2650 \mathrm{~m}$ environ. Sa pente moyenne avoisinait $45^{\circ}$ sur ces $250 \mathrm{~m}$ de hauteur, dont les $100 \mathrm{~m}$ inférieurs constituant une falaise plus raide $\left(60\right.$ à $\left.70^{\circ}\right)$ conférant au versant un aspect légèrement bombé. Cet ensemble se retrouve aujourd'hui situé entre les altitudes 2350 et 2600 , globalement inchangé même si peut-être légèrement déformé et/ou basculé,

La roche, dure, présente une structure subhorizontale à léger pendage Ouest c'est-à-dire rentrant dans le versant. La stratification est à peine visible, mais ressort bien sur certaines vues et garde sa continuité sur toute la largeur de la falaise. Quelques failles rayent le versant, qui ne paraissent pas avoir rejoué récemment : on les voit dès les premières photos aériennes (1948), on peut les retrouver ensuite lorsque l'angle de la prise de vues et l'éclairage sont favorables.

De près, la falaise apparaît très lézardée ; on ne s'étonne pas qu'il s'en soit détaché plusieurs pans de rocher instable. Le rocher est extrêmement fissuré dans le ressaut inférieur, à une maille décamétrique à métrique.

Le plus spectaculaire reste la transformation des couloirs, goulottes d'évacuation de débris de granulométries très variées, où il reste des accumulations instables de matériaux descendus de fraîche date (surtout abondantes dans les deux couloirs encadrants). On ignore l'effet exact de la neige et du gel sur l'évolution de ces tas de pierrailles.

\subsection{Autres versants}

L'examen des autres versants de la même montagne, au Nord au-dessus du lac de l'Etroit ou à l'Ouest en vue du Dos Rond, suggère qu'elle a été le siège de combinaisons analogues d'effondrements et de glisse- 


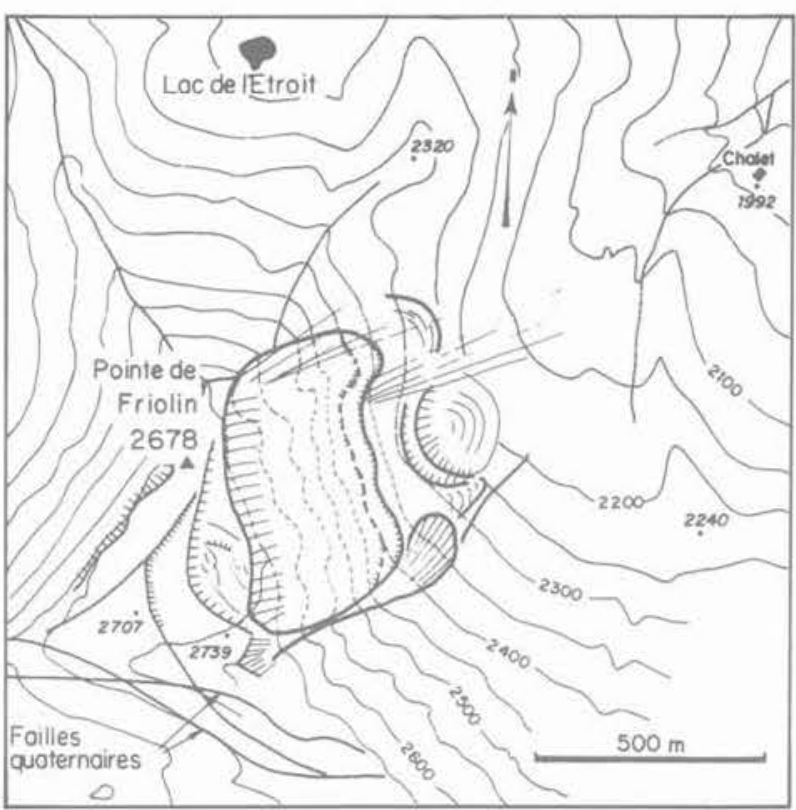

Fig. 8. - Plan d'ensemble.

Fig. 8. - General plan view

ments de ses flancs dans des temps très reculés (les terrains déplacés sont, à l'Ouest, en partie recouverts de moraines et de petits glaciers rocheux). Ces mouvements du sol ne sont jamais allés très loin.

Tout se passe comme si le Friolin a implose » par crises successives depuis une époque très éloignée, du fait de la présence de gypse soluble en dessous de lui. Nul doute qu'il y aura d'autres manifestations de cette instabilité structurale. (Remarques de A. PACHOUD).

\subsection{Hydrologie}

A Peisey (alt. $1300 \mathrm{~m}$ ), où elles sont régulièrement mesurées depuis 30 ans, les précipitations totales tournent entre 700 et $1200 \mathrm{~mm}$ par an, avec un maximum bien détaché de $1331 \mathrm{~mm}$ en 1981 .

On ne connaît pas directement leur nature (pluie ou neige), ni leurs variations avec l'altitude. La forte valeur de 1981 tient aux mois de septembre et octobre ( $338 \mathrm{~mm}$ contre 148 en moyenne pour ces deux mois là), et au mois de décembre (maximum trentennaire lui aussi, avec $272 \mathrm{~mm}$ contre 97 en moyenne).

L'enneigement en altitude est régulièrement mesuré par EDF, qui procède tous les dix jours à des sondages pour déterminer la valeur en eau de la couche de neige en un certain nombre de stations. En Haute Isère, vers $2500 \mathrm{~m}$ d'altitude, les stocks accumulés durant les hivers 1980 et 1981 furent relativement exceptionnels : 1125 et $905 \mathrm{~mm}$ d'eau respectivement, contre $640 \mathrm{~mm}$ d'eau en moyenne. La fonte (qui intervient entre avril-mai et juin-juillet) libéra ces années-là jusqu'à $500 \mathrm{~mm}$ d'eau par mois, ce qui est une valeur élevée mais pas exceptionnelle.
L'année 1982, qui se distinguait par son très fort enneigement en janvier, n'eut en fin d'accumulation qu'un stock très moyen $(700 \mathrm{~mm})$. Les années 83 , 84 probablement, ainsi que 86 et 88 connurent à nouveau des enneigements importants en altitude en fin d'hiver.

On conçoit que, dans des conditions de forte perméabilité fissurale, seules des quantités d'eau très importantes puissent accroitre sensiblement les gradients hydrauliques moteurs ; elles sont surtout disponibles à la fonte (J.P. FOLLACCI, 1987). Au Friolin, les eaux infiltrées participent aussi à la déstabilisation du versant par la dissolution des gypses sous-jacents. Le résultat de cette activité souterraine de longue haleine a pu profiter, pour s'exprimer, de labondance des apports d'eau de 1980 et 1981. Mais le glissement ne s'est pas manifesté de façon très significative en 1986 et 88 .

\section{CONCLUSION}

De simples photos $24 \times 36$ prises avec un minimum de soin et depuis des points de vues bien repérés constituent un témoignage de premier ordre pour apprécier l'évolution d'un versant instable. Leur analyse requiert toutefois un travail approfondi.

Un glissement d'ampleur exceptionnelle s'est produit à partir de la fonte des neiges de 1982 au Friolin, dont le versant Est est descendu en quelques années de plus de $50 \mathrm{~m}$ sans se disloquer (les deux tiers du mouvement s'étant effectués semble-t-il en quelques mois). Cette montagne, qui culmine vers $2700 \mathrm{~m}$ d'altitude, est affectée par des dissolutions du gypse sous-jacent. Elle était observée depuis plus de 20 ans lorsque le mouvement se manifesta par d'abondantes chutes de blocs.

Le volume déplacé avoisinerait 10 millions de $\mathrm{m}^{3}$. A-t-il trouvé une butée de pied suffisante, ou doit-on craindre une reprise du mouvement? Quelle forme pourrait-elle prendre? Il ne faudrait pas relâcher la vigilance, sous prétexte que le mouvement s'est nettement ralenti après quelques années. Il y a lieu de se demander comment le versant glissé tient encore, sachant que toute la surface rompue a perdu sa cohésion et se trouve certainement en état de frottement résiduel ; et de s'interroger sur les tolérances d'adaptation de la masse glissée, qui ne semble butée à son pied que d'un côté.

L'analyse photogrammétrique des prises de vues systématiques de l'IGN pourrait fournir une cinématique complète du mouvement entre 1970, 1980, 1982 et 1986. On espère ainsi éclairer l'appréciation du risque d'un éboulement majeur, avant de réfléchir aux dispositions de surveillance et d'auscultation les plus appropriées.

\section{REMERCIEMENTS}

Je dédie cette étude à la mémoire de mon père, décédé début 87 , qui m'a initié là-haut à la Géologie de l'Ingénieur et à l'Auscultation.

Le Bureau COYNE et BELLIER a pris en charge la mise en forme et la diffusion de ce travail. Qu'il en 
soit remercié, ainsi que la Division Technique Générale d'EDF pour ses précieux renseignements hydrologiques, et les bergers de Peisey pour leurs témoignages irremplaçables.

\section{PHOTOGRAPHIES AÉRIENNES IGN CONSEILLEES}

Bourg-St-Maurice - Moutiers/3532-3533 $\mathrm{n}^{\circ} 160$ et 161

$1970 \quad 3533-3633 / 300 n^{\circ} 255$ et 257

$1980 \quad n^{\circ} 81$ et 82

$1982 \quad n^{\circ} 1315$ et 1316

1986 Moutiers - Tignes/300/3533-3633/

$n^{\circ} 144$ et 146

\section{BIBLIOGRAPHIE}

ANTOINE P., CAMPOROTA P., GIRAUD A., ROCHET L. (1987), La menace d'écroulement aux Ruines de Séchilienne (Isère). Bull. Liaison Labo P. et Ch. - 150/151 - j.a/s.o 1987, p. 55-64.

FOLLACCI J.P. (1987), Les mouvements du versant de la Clapière à Saint-Etienne-de-Tinée (AlpesMaritimes). Bull. Liaison Labo P. et Ch. $-150 / 151$ - j.a/s.o 1987 , p. 39-54.
GOGUEL J. (1969), Des failles quaternaires à Peisey (Savoie). Bull. Soc. Géol. de France (7), XI, p. 35-39.

GOGUEL J. et HUMBERT M. (1972), Carte des risques géologiques pour la commune de Peisey Nancroix. Essai méthodologique BRGM.

GOGUEL J. et PACHOUD A. (1972), Géologie et dynamique de l'écroulement du mont Granier dans le Massif de la Chartreuse en 1248. Bull. BRGM, Section III, $n^{\circ} 1,1972$, p. 29-38.

GOGUEL J. (1980), Géologie de l'environnement, Masson, Paris, 190 p. (chapitre 3 - Glissements et éboulements, p.29-44).

GOGUEL J. (1980), Les risques de grands éboulements. La Recherche $N^{\circ} 111$, mai 1980, p. 620-628.

GOGUEL J. et PACHOUD A. (1982), Examen géologique des chutes de blocs de la Pointe de Frio. lin. Rapport BRGM pour la Direction Départementale de la Sécurité Civile.

GUILLOT F. (1987), Géologie de lantépermien de Vanoise Septentrionale. Thèse présentée à l'UST de Lille Flandres Artois, soutenue le 24 mars 1987, pour obtenir le titre de Docteur de lUniversité.

VOIGHT B. (Ed.) (1978-1979), Rockslides and avalanches, 1, Elsevier Sc. P. Cy, Amsterdam Oxford - N.Y., 833 p.

\section{INDICATIONS PRATIQUES \\ pour la visite du FRIOLIN :}

- saisir une belle journée d'août ou septembre, et partir très tôt sous peine de trouver le versant Est dans l'ombre et les nuées déjà formées ;

- accès commodes au plateau sommital par le Sud-Ouest (en montant de La Plagne) ou le Sud-Est (en descendant de Bellecôte), ainsi que par l'arête Nord-Ouest (aisément accessible depuis le lac de l'Etroit, au-dessus de Peisey, et bien praticable) ;

- il est formellement déconseillé d'emprunter l'arête Nord-Est, localement très dangereuse (sinon même impraticable à la montée) ;

- de Peisey-Nancroix, par le chemin qui part du camping (1 $200 \mathrm{~m}$ de dénivellation), et à condition d'avoir le pied sûr, on aura avantage à prendre du recul au dessus des Grandes Baraques pour voir le versant Est dans son ensemble depuis le point 2303 avant d'aborder la Montagne de Bellecôte ou le collet proche du sommet Sud du Friolin (2 739) par un couloir herbu raide un peu délicat. 